\title{
Eventos Extremos de Chuva no Estado do Rio Grande do Sul, Brasil, entre 2004 e 2013. Parte 1: Definição dos Eventos e Estatísticas
}

\author{
Mateus da Silva Teixeira ${ }^{1}$ (D) , Raidel Báez Prieto $^{2}$ \\ ${ }^{1}$ Faculdade de Meteorologia, Universidade Federal de Pelotas, Pelotas, RS, Brasil. \\ ${ }^{2}$ Universidade Estadual de Santa Catarina, Florianópolis, SC, Brasil.
}

Recebido em: 18 de Fevereiro de 2019 - Aceito em: 2 de Agosto de 2019

\begin{abstract}
Resumo
Este artigo avaliou os eventos de chuva extrema ocorridos entre 2004 e 2013 no estado do Rio Grande do Sul, Brasil. Os eventos extremos foram separados em duas classes: diários e persistentes, os quais foram identificados por meio das estações meteorológicas do INMET. Para ambas classes de eventos, nenhuma tendência de aumento ou diminuição no número de casos foi detectada. Os eventos extremos diários têm maior frequência na primavera e no verão, enquanto os eventos extremos persistentes têm maior frequência no inverno. A espacialização da chuva, nos casos extremos persistentes, mostrou que a metade leste do estado do Rio Grande do Sul tem maior frequência de eventos de chuva persistente, com grande quantidade de chuva. Em um segundo artigo, os padrões atmosféricos são investigados, bem como a sua relação com a espacialização observada da chuva, obtida neste primeiro artigo.
\end{abstract}

Palavras-chave: chuva persistente, espacialização chuva, quantis, tendências.

\section{Extreme Rainfall Events in the state of Rio Grande do Sul, Brazil, Between 2004 and 2013. Part 1: Events Definition and Statistics}

\begin{abstract}
This article evaluated the extreme rainfall events occurred between 2004 and 2013 in the state of Rio Grande do Sul, Brazil. The extreme events were separated into two groups: daily and persistent events, which were identified with meteorological stations from INMET. For both classes of events, any trend of increase or decrease in the number of events was detected. The daily extreme events have higher frequency in spring and summer, while the persistent extreme events have higher frequency in winter. Spatialisation of rainfall, of persistent extreme events, showed that the east half of state of Rio Grande do Sul has a higher frequency of persistent rainfall events with larger rainfall quantities. In a second article, the atmospheric patterns will be investigated, as well as their relationships with the observed rainfall spatialisation, obtained in this first article.
\end{abstract}

Keywords: persistent rainfall, rainfall spatialization, quantiles, trends.

\section{Introdução}

Nos últimos anos os eventos de chuva intensa têm se tornado cada vez mais preocupantes, especialmente pelos danos socioeconômicos decorrentes. Estudos anteriores (Teixeira e Satyamurty, 2011; Nunes e Da Silva, 2013) mostraram a existência de aumento no número de eventos de chuva intensa na região sul do Brasil e projeções de aumento desses eventos no futuro. Além do próprio fenômeno meteorológico, alguns fatores geoambientais podem colaborar para aumentar o potencial de perigo relacionado à chuva: grande quantidade de rios; topografia acidentada; crescimento e ocupação populacionais desordenados.

Diferentemente das demais regiões brasileiras, a região sul do Brasil não possui sazonalidade destacada em relação ao comportamento pluviométrico (Rao e Hada, 1990; Barbieri, 2005; Franchito et al., 2008), quando comparada a outras regiões brasileiras. Há, sim, uma aproximada uniformidade na distribuição da chuva ao longo do ano. Portanto, há uma consequente maior probabilidade de ocorrência de chuvas extremas em qualquer época do ano

Autor de correspondência: Mateus da Silva Teixeira, mateus.teixeira@ufpel.edu.br. 
na região sul do Brasil, em comparação à região sudeste, por exemplo, que possui uma probabilidade de eventos de chuva extrema muito menor no período do inverno - seu conhecido período mais seco.

O exposto acima evidencia a importância da previsão de tempo de boa qualidade. Entretanto, a previsão quantitativa de precipitação é uma das tarefas mais complicadas, atualmente, da Meteorologia (Doswell III et al. 1996). Eventos extremos de chuva dependem fortemente do aporte de umidade, do grau de instabilidade atmosférica presente e de particularidades cinemáticas dos fenômenos causadores da chuva, como velocidade de deslocamento dos sistemas, a sua orientação em relação a sua velocidade de deslocamento, por exemplo.

A destreza da previsão meteorológica destes eventos depende fortemente de um bom conhecimento da dinâmica atmosférica e dos processos meteorológicos responsáveis por estes eventos. A forte relação negativa (estatisticamente significativa) entre o Índice de Oscilação Sul (IOS) e a chuva nesta região do Brasil, especialmente na primavera austral (Ropelewski e Halpert, 1989; Rao e Hada, 1990; Grimm et al., 2000), é um exemplo do benefício à previsão do tempo que se consegue ao se entender melhor os fenômenos envolvidos no comportamento da chuva, pois em eventos El-Niño (IOS negativo) espera-se maiores quantidades de chuva no sul do Brasil.

Vários autores estudaram eventos de chuva intensa em diferentes regiões do mundo, inclusive no Brasil (Severo, 1994; Junker et al., 1999; Harnack et al., 1999; Teixeira e Satyamurty, 2007; Lima et al., 2010; Nunes e Da Silva, 2013, por exemplo). Em comum a estes estudos está a obtenção das configurações atmosféricas, em escala sinótica, associadas a eventos de chuva intensa.

Tang et al. (2006), Chen e Zhai (2013) e Zhao et al. (2017) estudaram eventos persistente de chuva ocorridos na China. Diferente da região sul do Brasil, a China possui um período mais provável para a ocorrência de chuvas persistentes, se estendendo de abril a julho, durante o verão do hemisfério norte. Uma característica comum aos eventos estudados pelos autores é a maior ocorrência de chuva persistente próxima à costa do Mar da China, em especial o Meridional. Eles são mais raros na parte central e oeste do país.

Entretanto, até o momento pouca atenção tem sido dada aos eventos persistentes de chuva no Brasil. Esse tipo de evento pode provocar enormes danos à sociedade, uma vez que a chuva duradoura pode, mesmo em taxas diárias não consideradas intensas (abaixo de 40-50 $\mathrm{mm} \mathrm{dia}^{-1}$ ), levar a acumulados de água muito elevados, acarretando saturação do solo, deslizamento de terra, elevação de rios, por exemplo. Além disso, conforme já enfatizado, a região sul do Brasil pode apresentar eventos importantes de chuva em qualquer época do ano.

Prieto e Teixeira (2016) investigaram um caso de chuva persistente ocorrido em maio de 2004, no estado do
Rio Grande do Sul (RS), no qual várias estações meteorológicas localizadas na costa do estado registraram chuvas superiores a $200 \mathrm{~mm}$, durante 6 dias.

Este trabalho, portanto, foca no estudo dos eventos extremos de chuva persistente, observados no estado do Rio Grande do Sul entre 2004 e 2013, comparando-os com os eventos extremos diários de chuva. Aqui é apresentada a primeira parte dessa investigação, onde objetiva-se definir as duas classes de eventos de chuva estudados: (i) diário e (ii) persistente, e avaliar as suas estatísticas ao longo dos 10 anos de estudo. Numa segunda parte, serão apresentados os padrões atmosféricos associados aos dois tipos de eventos.

\section{Materiais e Métodos}

Os eventos de chuva extrema ocorridos no estado do Rio Grande do Sul foram investigados por meio dos dados pluviométricos diários provenientes do Banco de Dados Meteorológicos para Ensino e Pesquisa (BDMEP) do Instituto Nacional de Meteorologia (INMET). Um total de 18 estações meteorológicas foram usadas. A distribuição espacial destas estações meteorológicas é apresentada na Fig. 1. As normais climatológicas de chuva para estas estações e para o período 1981-2010 foram usadas na definição de chuva extrema diária.

Uma avaliação subjetiva da relação entre a frequência anual de casos de chuva persistente e as fases do fenômeno El Niño Oscilação Sul (ENOS) é realizada usando o Ocean Index Niño (ONI), calculado pelo Climate Prediction Center do National Centers for Environmental Prediction (CPC/NCEP).

A definição de um evento de chuva extrema é altamente subjetiva, podendo ser estabelecida levando-se em conta aspectos como: intensidade, a quantidade total registrada num certo intervalo de tempo e abrangência espacial, bem como quaisquer combinações destas características da chuva. Neste estudo os eventos de chuva extrema foram divididos em duas categorias principais: (i) diários e (ii) persistentes. Os eventos extremos diários são aqueles eventos em que uma grande quantidade de chuva foi registrada em apenas 24 horas. A expressão "grande quantidade de chuva" é determinada, neste estudo, como chuva superior a $20 \%$ do total sazonal climatológico (Carvalho et al. 2002). Já os eventos extremos persistentes são aqueles eventos com grande quantidade de chuva, extensa duração (em dias) e abrangência (em número de estações meteorológicas). Os passos abaixo foram usados para definição e identificação dos eventos persistentes, seguindo aqueles usados por Prieto e Teixeira (2016), diferindo apenas no limiar de chuva acumulado:

1. Contabilização dos dias consecutivos com chuva (mais de um dia), sendo cada período consecutivo chamado de período de chuva e obtenção do acumulado de 


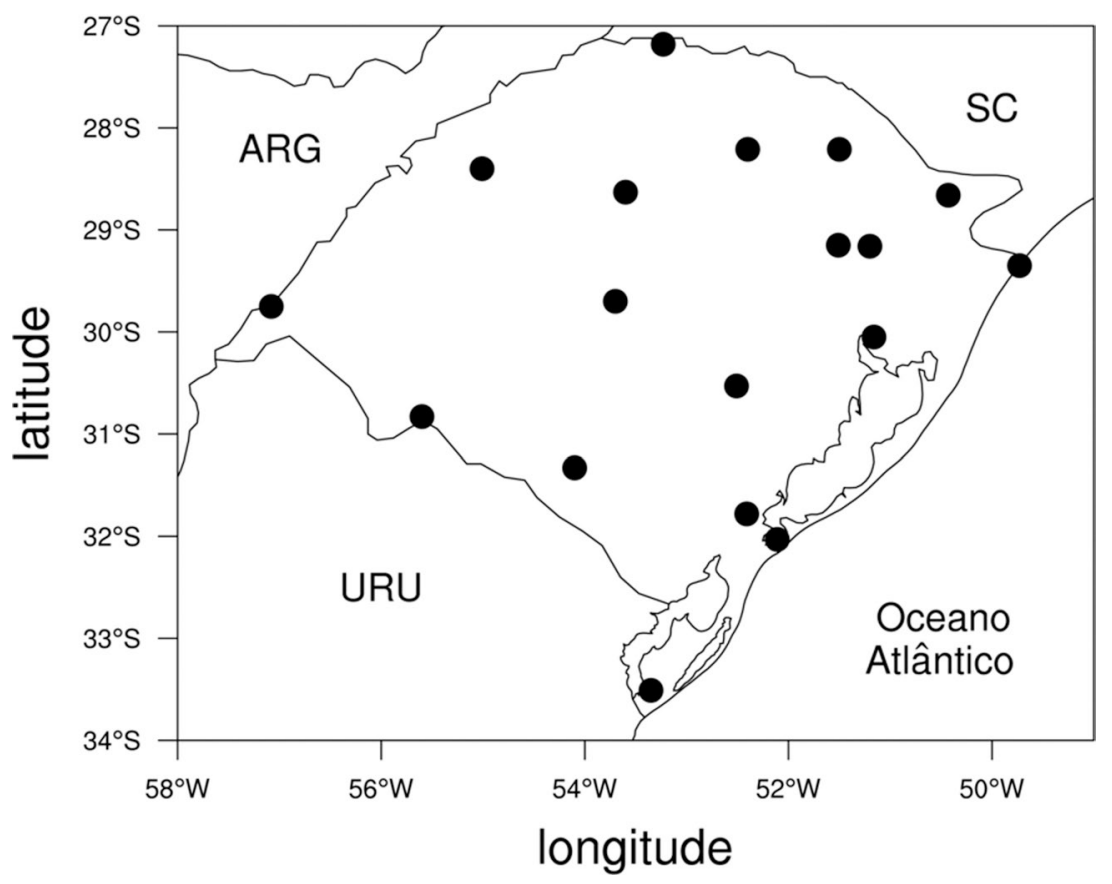

Figura 1 - Localização geográfica das 18 estações meteorológicas convencionais do BDMEP/INMET existentes no estado do Rio Grande do Sul.

chuva para cada período, para cada estação meteorológica.

2. Identificação do número de estações meteorológicas atingidas pelos períodos identificados no passo (1) e determinação dos eventos de chuva persistente: duração e abrangência. Nesse passo, é realizada uma avaliação da intersecção dos períodos de chuva detectados em cada uma das 18 estações meteorológicas (passo 1). A intersecção dos períodos de chuva permite determinar o início e o fim da chuva persistente, possibilitando a definição dos eventos de chuva persistente. Cria-se, portanto, um banco de dados contendo a duração (quantidade de dias) dos eventos, a quantidade máxima de estações meteorológicas que registraram chuva durante o evento e o total acumulado de chuva no período, para cada estação meteorológica.

3. Seleção dos casos finais de chuva persistente que atendem os seguintes critérios:

a. Duração (dias) superior ou igual ao quantil de $90 \%$ das durações de todos os períodos de chuva;

b. Abrangência espacial de no mínimo 5 estações meteorológicas (equivalente a aproximadamente $30 \%$ do total de estações meteorológicas) com acumulados superiores a $60 \%$ da média climatológica sazonal, baseada nas normais climatológicas do INMET para o período 1981-2010.

A definição de evento persistente deste trabalho vai ao encontro daquelas estabelecidas por Tang et al. (2006) e Chen e Zhai (2013), por exemplo, que não usam apenas um critério. Entretanto, difere-se substancialmente, pois permite uma aplicação mais geral por levar em conta o caráter estatístico e climatológico dos eventos. Em suma, a definição aqui proposta não determina quantidades fixas para duração da chuva e para chuva acumulada, deixando que sejam estabelecidas em função do comportamento pluviométrico da região de interesse, uma vez que é esperado que este varie consideravelmente de uma região para outra.

A existência de uma tendência no número de eventos ao longo do período estudado foi verificada por meio do teste de Mann-Kendall, um teste não paramétrico que permite identificar se uma série temporal possui alguma tendência.

\section{Resultados e Discussões}

A Fig. 2 mostra o diagrama de dispersão Duração do evento vs. Chuva total para todos os casos de chuva identificados. Na abscissa pode-se identificar os casos em função da sua duração. Por exemplo, a duração de oito dias de um caso significa que durante oito dias foi detectado chuva em ao menos 5 estações meteorológicas. No dia anterior e posterior a esse período, nenhuma estação meteorológica apresentou chuva. $\mathrm{Na}$ ordenada pode-se identificar os casos em função da chuva total, que nesse caso se refere à chuva acumulada em todo o período de chuva do caso, média entre todas as estações meteorológicas. As linhas horizontais e verticais identificam quantis específicos para cada uma das informações dos eventos. As linhas cinzas e contínuas identificam a mediana, ou quantil de $50 \%$, enquanto as linhas pretas e tracejadas identificam o quantil extremo de $90 \%$. Desta forma, neste trabalho, os casos de chuva persistente severa foram considerados como aqueles 


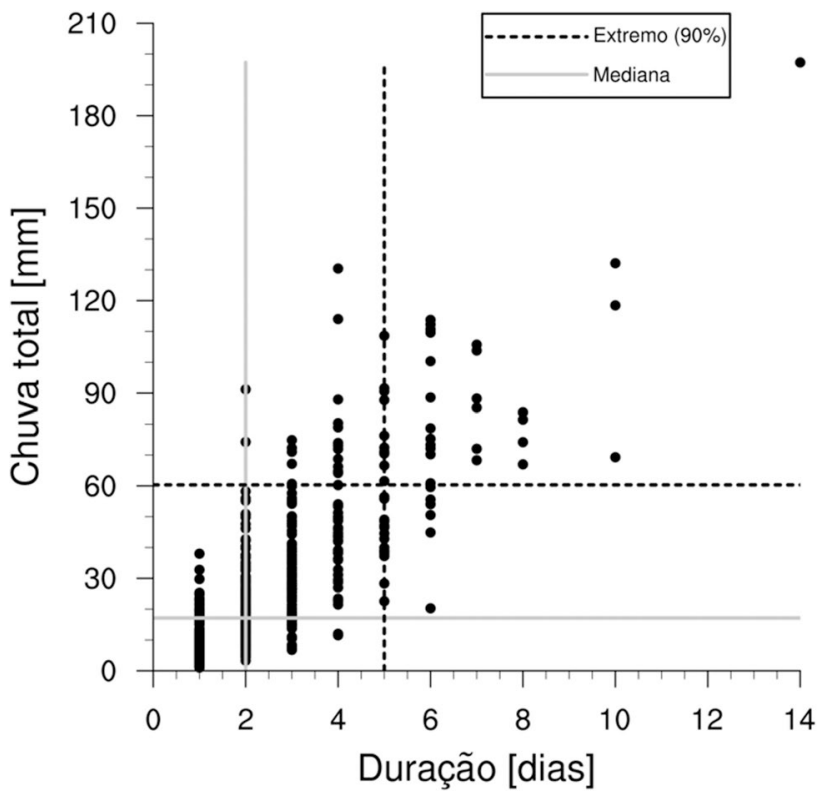

Figura 2 - Dispersão entre a duração da chuva (em dias) e o total de chuva acumulado observado (em $\mathrm{mm}$ ).

concentrados à esquerda e acima das linhas vermelhas, ou em outras palavras, um caso de chuva é considerado persistente e extremo quando ele durar 5 ou mais dias e gerar um acumulado total igual ou superior a $60 \mathrm{~mm}$. Portanto, foram identificados um total de 37 casos de chuva persistente no estado do RS.

A Fig. 3 mostra as frequências mensal e anual para os 37 casos identificados de chuva persistente. Pode-se perceber que o mês de fevereiro se destaca em relação aos demais quanto à quantidade de casos persistentes. Outros

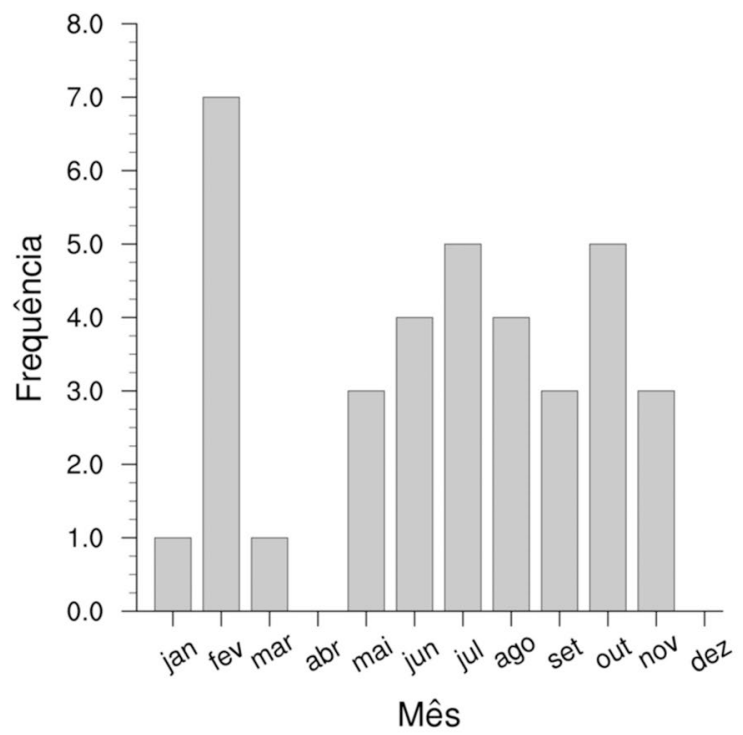

(a) dois máximos podem ser observados: no inverno (em julho) e na primavera (em outubro). A estação do outono se destaca como aquela com menor quantidade de casos persistentes de chuva, não tendo nenhum sido identificado no mês de abril. A variação anual dos casos de chuva persistente mostra uma oscilação em torno de aproximadamente 3 casos por ano. Os anos de 2005, 2009 e 2011 se destacam com as maiores frequências de casos de chuva persistente, enquanto que os anos de 2006 e $2010 \mathrm{com}$ as menores frequências. O curto período de análise deste trabalho não permite que quaisquer conclusões importantes sejam obtidas em relação à ocorrência desses casos e uma influência das fases do fenômeno El Niño Oscilação Sul (ENOS), mas pode-se perceber que não há uma possível relação entre casos de chuva persistente e o ENOS. Por exemplo, os períodos de 2007-2008 e 2009-2010 são classificados como La Niña e El Niño, respectivamente, mas apresentam frequências similares dos casos de chuva persistente. Obviamente, um período maior precisa ser investigado para uma conclusão mais robusta.

Na Fig. 2 pode-se perceber que um grupo de casos tiveram apenas um dia de duração. Esse grupo compõe os eventos diários de chuva. Os eventos severos foram selecionados quando a chuva diária de ao menos duas estações meteorológicas ultrapassou $20 \%$ do total sazonal climatológico (de cada estação meteorológica). Esse critério permite descartar casos que podem ter sido causados por condições estritamente locais, em que a condição sinótica não necessariamente tenha tido um papel importante. É importante enfatizar que os casos discriminados como diários são caracterizados por terem chuva significativa em apenas um dia, ou seja, não apresentam chuva importante nem antes e nem depois.

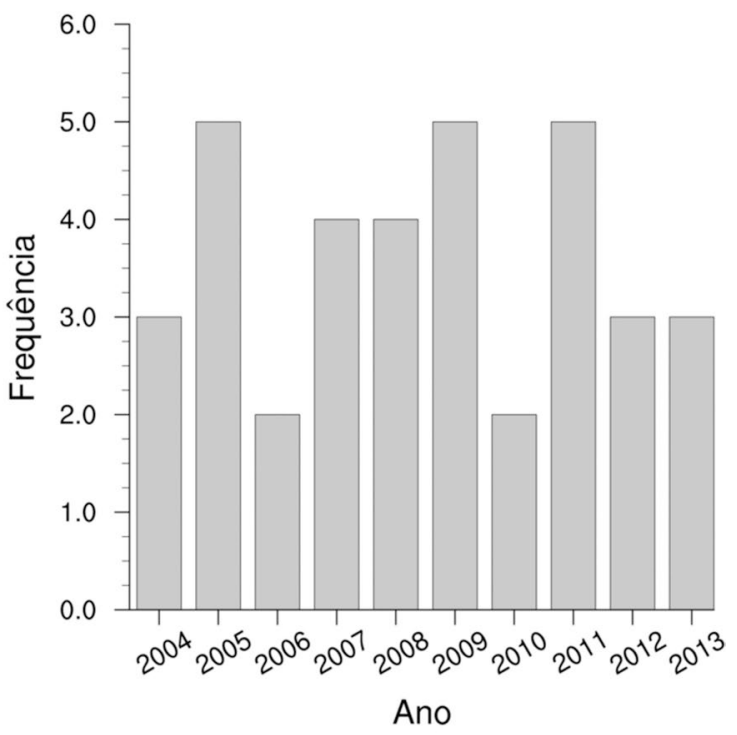

(b)

Figura 3 - Frequências mensal (a) e anual (b) dos casos de chuva persistente no estado do RS. 
A Fig. 4 apresenta as frequências mensais e anuais dos casos diários de chuva. Na distribuição mensal dos casos é possível destacar os períodos da primavera (SON) e verão (JFM) como mais propensos a casos de chuva severa diária, visto que os meses de dezembro e janeiro se destacam. O outono também se destaca, com abril possuindo a maior frequência de casos diários de chuva entre todos os meses do ano. Por outro lado, os meses de fevereiro e março se destacam com as menores quantidades de casos.

A distribuição anual apresenta uma oscilação dos casos ao longo dos 10 anos do estudo. Destacam-se os anos de 2006, 2008, 2009 e 2012, com 9 ou mais casos, e também os anos de 2005, 2007 e 2010, todos com apenas 4 casos. Pelos mesmos motivos descritos para os casos de chuva persistente, não há como estabelecer relação entre as fases do ENOS e esses casos de chuva diária, pois frequências de casos ocorrem tanto em anos com fase fria do ENOS (2008 e 2012), quanto durante a fase quente do ENOS (2009).

Durante os dez anos de avaliação deste estudo não foi possível identificar quaisquer tendências nas frequências anuais dos eventos de chuva, tanto persistentes quanto diários, por meio do teste de Mann-Kendall, ou seja, não há tendências significativas de aumento ou diminuição no número de eventos (diários ou persistentes) ao longo dos anos estudados.

A Tabela 1 apresenta a distribuição sazonal dos eventos de chuva persistente separados por sua duração. Dessa tabela pode-se perceber que eventos com 5 e 6 dias de duração foram mais comuns durante o período estudado. Esse resultado é esperado, uma vez que o limite inferior de seleção foi de cinco dias e que a raridade de um

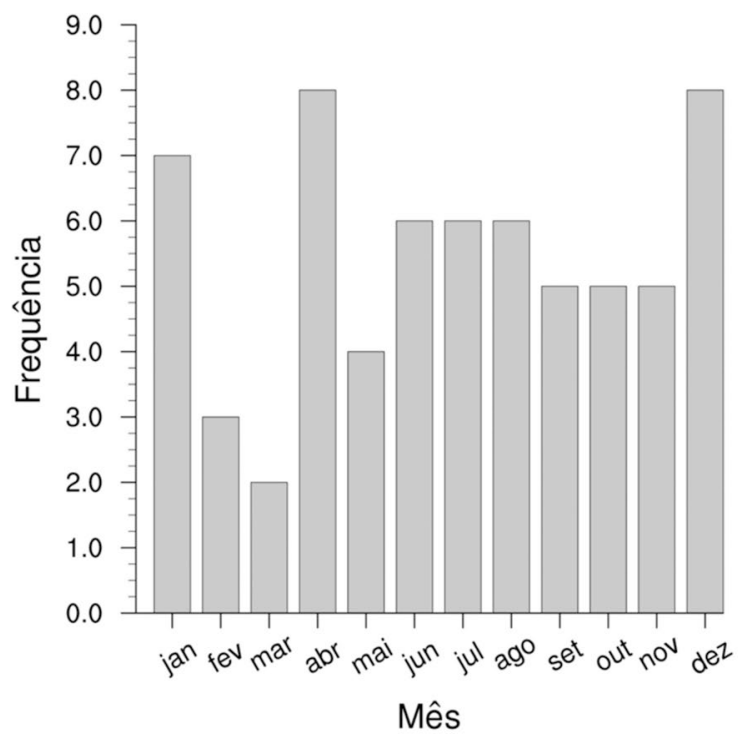

(a)
Tabela 1 - Distribuição sazonal dos casos de chuva persistentes em função da duração, em dias.

\begin{tabular}{lccccc}
\hline Duração & JFM & AMJ & JAS & OND & Total \\
\hline 5 & 1 & 2 & 5 & 2 & 10 \\
6 & 5 & 0 & 7 & 2 & 14 \\
7 & 2 & 1 & 2 & 1 & 6 \\
8 & 0 & 2 & 0 & 1 & 3 \\
10 & 1 & 2 & 0 & 0 & 3 \\
14 & 0 & 0 & 0 & 1 & 1 \\
Total & 9 & 7 & 14 & 7 & 37 \\
\hline
\end{tabular}

evento prolongado de chuva é diretamente proporcional ao número de dias consecutivos de chuva. Ou seja, os eventos mais raros são aqueles que duram muitos dias. $\mathrm{O}$ evento que durou 14 dias é um exemplo, que além de uma grande duração, teve a maior média de chuva: quase $200 \mathrm{~mm}$. Outro ponto interessante, mas também esperado, está na estação de maior ocorrência: o inverno (JAS), com 14 eventos. Nas demais estações, a frequência dos eventos é similar. Andrade (2005) e Cavalcanti e Kousky (2009) mostraram que os meses de julho a outubro possuem as maiores frequências mensais de passagem de sistemas frontais no sul do Brasil. Por essa frequência mais elevada nesse período é esperada uma maior probabilidade de frentes estacionárias sobre a região de estudo, levando a períodos prolongados de chuva.

A espacialização da chuva, bem como o seu grau de intensidade, pode ser visualizada nos gráficos da Fig. 5. Nesses gráficos são apresentadas as porcentagens de eventos que provocaram chuva superior a $60 \%$ da média sazonal (um dos critérios de seleção dos eventos persistentes)

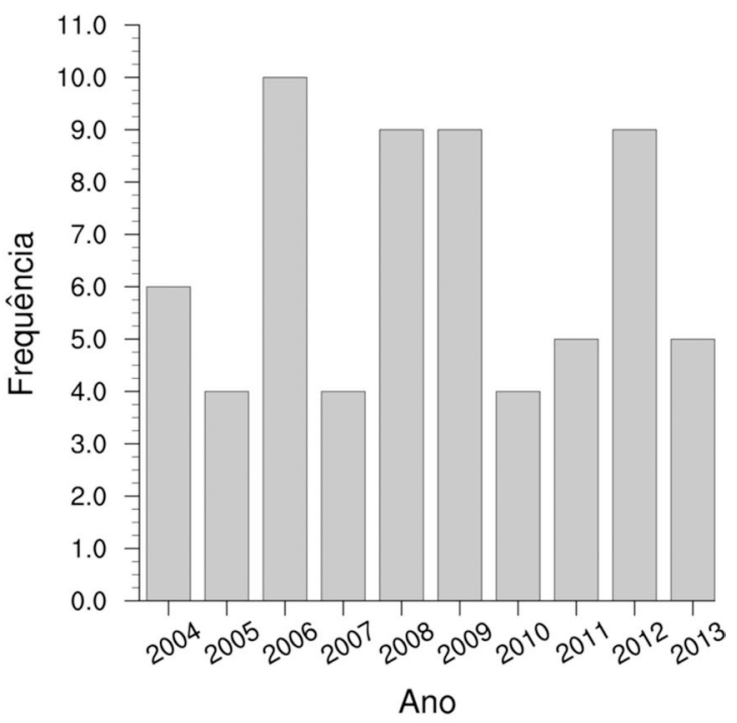

(b)

Figura 4 - Frequências mensal (a) e anual (b) dos casos de chuva intensa diária. 


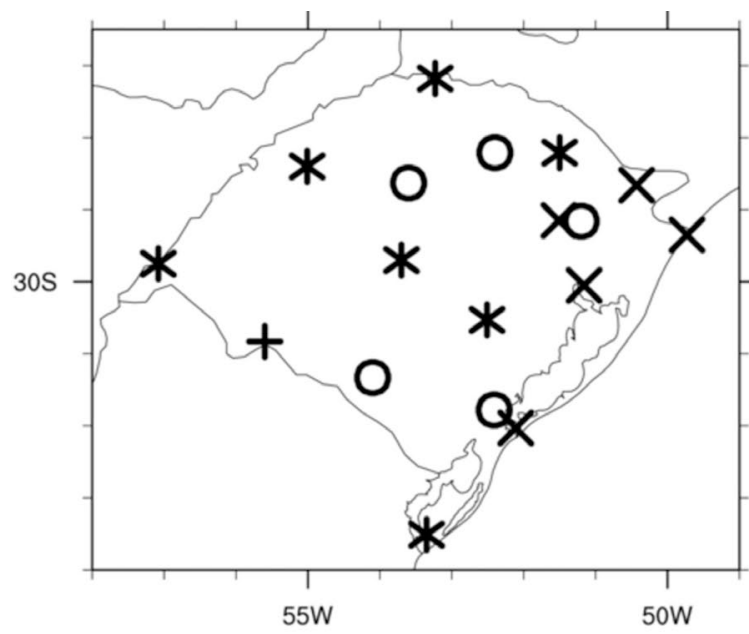

(a)

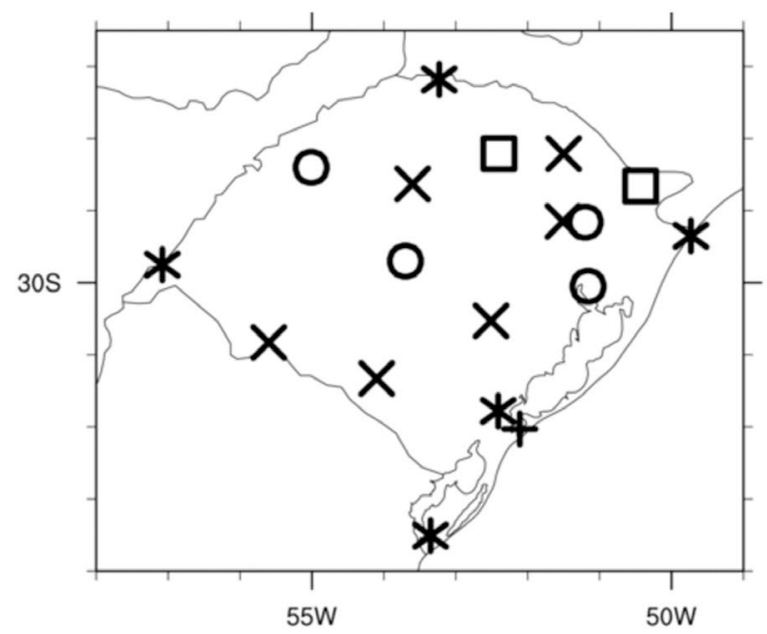

(c)

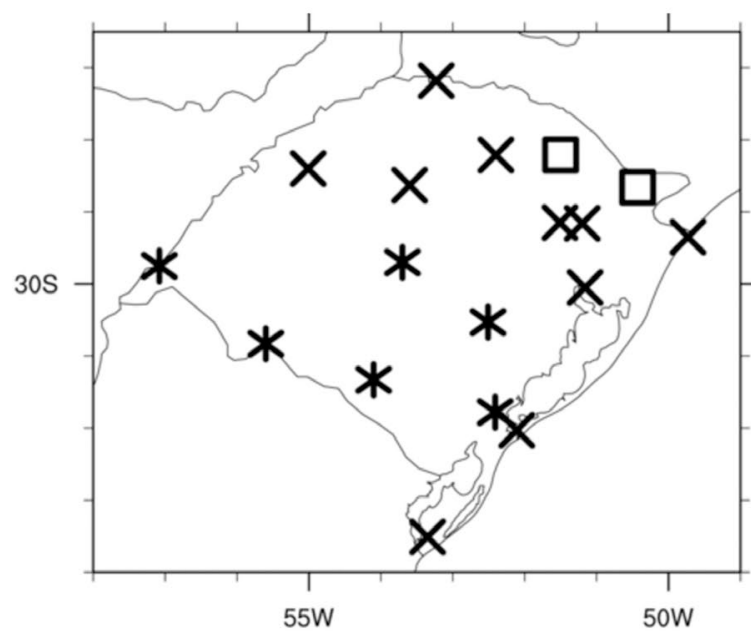

(e)

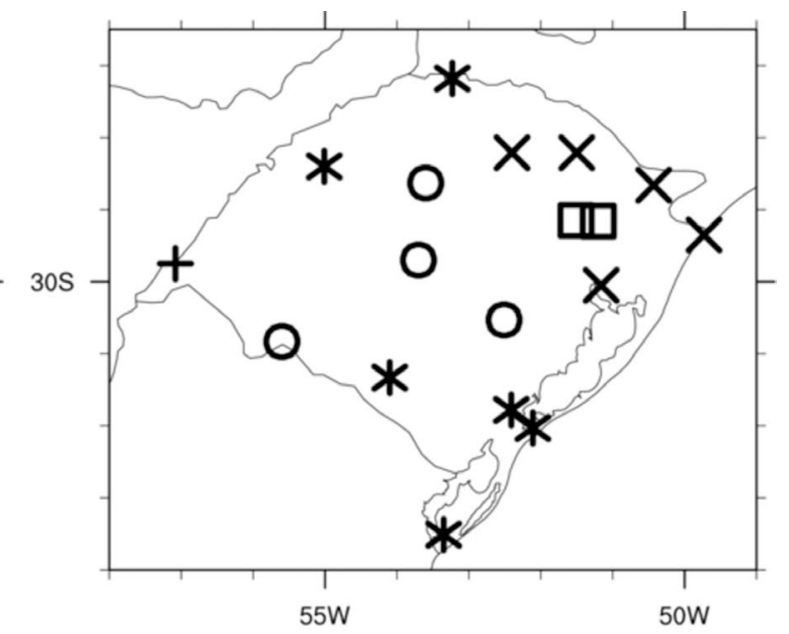

(b)

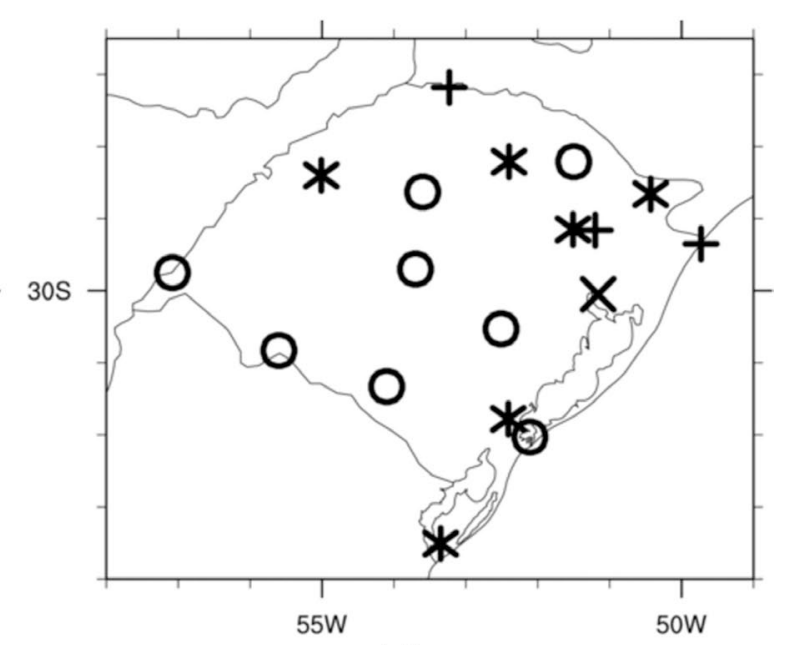

(d)

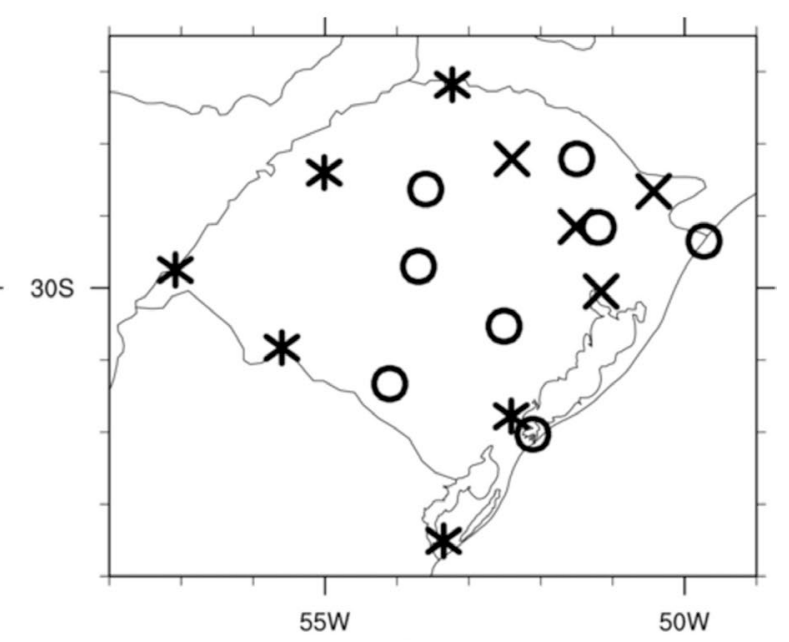

(f)

\section{$+[0-20) \quad *[20-40) \quad 0[40-60) \times[60-80) \quad \square[80-100]$}

Figura 5 - Porcentagens de casos de chuva persistente que provocaram chuva superior ou igual a $60 \%$ da média sazonal. Cada mapa refere-se à duração dos eventos: (a) 5 dias, (b) 6 dias, (c) 7 dias, (d) 8 dias e (e) 10 dias. O mapa em (f) refere-se a todos os eventos. 
em cada uma das 18 estações meteorológicas. Pode-se perceber que a maior parte dos eventos - superior a $60 \%$ provocaram chuvas acima desse limiar nas regiões nordeste, parte da região norte e da parte da região metropolitana de Porto Alegre, independente da duração do evento. No conjunto de casos que duraram de 6 a 8 dias, boa parte dos eventos - de 40 a $60 \%$ - provocaram chuva elevadas nas regiões central e sudoeste do RS, também (Figs. 5b-d). Nos casos com 10 dias de duração, grande parte dos casos (superior a 60\%) levaram chuvas fortes em praticamente todo o estado, exceto na região sudoeste. Quando se avalia todos os eventos (Fig. 5f), independente da sua duração, pode-se perceber que o extremo sul do RS e a sua parte oeste têm porcentagem menor de casos que levaram chuvas fortes. Então, é possível concluir que a metade leste do RS é, geralmente, a mais afetada pelos eventos de chuva persistente, em comparação às demais regiões. Esse resultado concorda com aqueles de Tang et al. (2006) e Chen e Zhai (2013) que também encontraram maior ocorrência dos eventos persistentes no leste da China. Na segunda parte deste trabalho, a ligação dessa espacialização com os padrões atmosféricos é descrita e analisada.

\section{Conclusões}

A partir dos resultados obtidos, pode-se concluir que:

- Os eventos extremos de chuva persistentes - aqueles que duram 5 ou mais dias e geram um acumulado total igual ou superior a $60 \mathrm{~mm}$ - podem durar até 14 dias, sendo mais frequentes aqueles com duração de 5 e 6 dias.

- Não foram identificadas quaisquer tendências de aumento ou diminuição na quantidade anual dos casos de chuva persistente e diária.

- Não há como identificar uma relação entre os casos de chuva (diária e persistente) com as fases do fenômeno ENOS. Uma quantidade de anos maior poderia melhorar a avaliação dessa relação.

- As estações do inverno e da primavera são aquelas em que os casos de chuva persistente ocorrem com maior frequência. Uma possível explicação para isso pode ser a maior suscetibilidade de sistemas frontais semi-estacionários. Na estação do inverno e primavera, há uma frequência maior de ocorrência de bloqueios nos Oceanos Pacífico e Atlântico - a oeste e a leste da América do Sul (Marques, 1996; Marques e Rao, 1999). A ocorrência de bloqueios nessas áreas pode colaborar para um deslocamento muito lento dos sistemas meteorológicos sobre a região do RS, levando a um prolongamento no tempo de atuação, que pode levar a chuvas persistentes e a grandes acúmulos de chuva.

- A metade leste do estado do RS é mais afetada pelos eventos extremos de chuva persistente. A razão disso será investigada na segunda parte deste estudo.

\section{Referências}

ANDRADE, K.M. Climatologia e Comportamento dos Sistemas Frontais Sobre a América do Sul. Dissertação de Mestrado em Meteorologia, Instituto Nacional de Pesquisas Espaciais, São José dos Campos, 79 p, 2005.

BARBIERI, P.R.B. Caracterização da Estação Chuvosa nas Regiões Sul e Sudeste do Brasil Associado com a Circulação Atmosférica. Dissertação de Mestrado, Instituto Nacional de Pesquisas Espaciais, São José dos Campos, São Paulo, Brasil, 2005.

CARVALHO, L.M.V.; JONES, C.; LEIBMANN, B. Extreme precipitation events in southeastern South America and large-scale convective patterns in the South Atlantic convergence zone. Journal of Climate, v. 15, n. 17, p. 23772394, 2002.

CAVALCANTI, I.F.A.; KOUSKY, V.E. Frentes frias sobre o Brasil. In: CAVALCANTI, I.F.A.; FERREIRA, N.J.; SILVA DIAS, M.A.F.; JUSTI DA SILVA, M.G.A. (org.). Tempo e Clima no Brasil. Oficina de Textos, São Paulo, p. 135-147, 2009.

CHEN, Y.; ZHAI, P. Persistent extreme precipitation events in China during 1951-2010. Climate Research, v. 57, n. 2, p. 143-155, 2013.

DOSWELL, C.A.; BROOKS, H.E.; MADDOX, R.A. Flash flood forecasting: An ingredients-based methodology. Weather and Forecasting, v. 11, n. 4, p. 560-581, 1996.

FRANCHITO, S.H.; RAO, V.B.; BARBIERI, P.R.B; SANTO, C.M. Rainy-season duration estimated from OLR versus rain gauge data and the 2001 drought in Southeast Brazil. Journal of Applied Meteorology and Climatology, v. 47, n. 5, p. 1493-1499, 2008.

GRIMM, A.M.; BARROS, V.R.; DOYLE, M.E. Climate variability in southern south america associated with el-niño and la-niña events. Journal of Climate, v. 13, n. 1, p. 35-58, 2000.

HARNACK, R.P.; APEL, K.; CERMAK, J.R. Heavy precipitation events in new jersey: attendant upper air conditions. Weather and Forecasting, v. 14, n. 6, p. 933-954, 1999.

JUNKER, N.W.; SCHNEIDER, R.S.; FAUVER, S.L. A study of heavy rainfall events during the great mid-west flood of 1993. Weather and Forecasting, v. 14, n. 5, p. 701-712, 1999.

LIMA, K.C.; SATYAMURTY, P.; FERNÁNDEZ, J.P.R. Largescale atmospheric conditions associated with heavy rainfall episodes in southeast brazil. Theoretical and Applied Climatology, v. 101, n. 1-2, p. 121-135, 2010.

NUNES, A.B.; DA SILVA, G.C. Climatology of extreme rainfall events in Eastern and Northern Santa Catarina State: Present and future climate. Revista Brasileira de Geofísica, v. 31, n. 3, p. 413-425, 2013.

MARQUES, R.F.C. Bloqueio Atmosférico no Hemisfério Sul. Tese de Doutorado, Instituto Nacional de Pesquisas Espaciais, São José dos Campos, São Paulo, 1996.

MARQUES, R.F.C.; RAO, V.B. A diagnosis of a long-lasting blocking event over over the Southeast Pacific Ocean. Monthly Weather Review, v. 127, n. 8, p. 1761-1776, 1999.

PRIETO, R.B.; TEIXEIRA, M.S. Análisis sinóptico de um evento extremo y persistente de lluvia sobre el estado de 
Río Grande do Sul, Brasil, en mayo de 2004. Ciência e Natura, v. 38, n. 2, p. 1110-1115, 2016.

RAO, V.B.; HADA, K. Characteristics of rainfall over brazil: annual variations and connections with southern oscillation. Theorical and Applied Climatology, v. 42, n. 2, p. 81-91, 1990.

ROPELEWSKI, C.F.; HALPERT, M.S. Precipitation patterns associated with the high index phase of the southern oscillation. Journal of Climate, v. 2, n. 3, p. 268-284, 1989.

SEVERO, D.L. Estudo de Casos de Chuvas Intensas no Estado de Santa Catarina. Dissertação de Mestrado, Instituto Nacional de Pesquisas Espaciais, São José dos Campos, 1994.

TANG, Y.; GAN, J.; ZHAO, L.; GAO, K. On the climatology of persistent heavy rainfall events in China. Advances in Atmospheric Sciences, v. 23, n. 5, p. 678-692, 2006.

TEIXEIRA, M.S.; SATYAMURTY, P. Dynamical and synoptic characteristics of heavy rainfall episodes in southern Brazil. Monthly Weather Review, v. 135, n. 2, p. 598-617, 2007.
TEIXEIRA, M.S.; SATYAMURTY, P. Trends in the frequency of intense precipitation events in southern and southeastern brazil during 1960-2004. Journal of Climate, v. 24, n. 7, p. 1913-1921, 2011.

ZHAO, Y.; WANG, D.; LIANG, Z.; XU, J. On the dynamics of the large-scale circulation during persistent severe rainfall events in Southern China. Journal of the Meteorological Society of Japan, v. 95, n. 2, p. 111-125, 2017.

\section{Endereços de Internet}

Ocean Index Niño (ONI), Climate Prediction Center do National Centers for Environmental Prediction (CPC/NCEP), https://origin.cpc.ncep.noaa.gov/products/analysis_monitor ing/ensostuff/ONI_v5.php.

License information: This is an open-access article distributed under the terms of the Creative Commons Attribution License (type CC-BY), which permits unrestricted use, distribution and reproduction in any medium, provided the original article is properly cited. 